PROCEEDINGS OF THE

AMERICAN MATHEMATICAL SOCIETY

Volume 130, Number 7 , Pages 1893-1903

S 0002-9939(01)06289-X

Article electronically published on December 20, 2001

\title{
SET-THEORETIC COMPLETE INTERSECTIONS ON BINOMIALS
}

\author{
MARGHERITA BARILE, MARCEL MORALES, AND APOSTOLOS THOMA \\ (Communicated by Wolmer V. Vasconcelos)
}

\begin{abstract}
Let $V$ be an affine toric variety of codimension $r$ over a field of any characteristic. We completely characterize the affine toric varieties that are set-theoretic complete intersections on binomials. In particular we prove that in the characteristic zero case, $V$ is a set-theoretic complete intersection on binomials if and only if $V$ is a complete intersection. Moreover, if $F_{1}, \ldots, F_{r}$ are binomials such that $I(V)=\operatorname{rad}\left(F_{1}, \ldots, F_{r}\right)$, then $I(V)=\left(F_{1}, \ldots, F_{r}\right)$. While in the positive characteristic $p$ case, $V$ is a set-theoretic complete intersection on binomials if and only if $V$ is completely $p$-glued.

These results improve and complete all known results on these topics.
\end{abstract}

\section{INTRODUCTION}

The determination of the minimum number of equations needed to define an algebraic variety $V$ set-theoretically or ideal-theoretically is an old and important problem in Algebraic Geometry. In this paper we consider the case of toric varieties and it turns out that these two problems are strongly related in characteristic zero; see Theorem 4.

The ideal of a toric variety is a prime binomial ideal. A binomial ideal is an ideal minimally generated by binomials. Eisenbud and Sturmfels began the systematic study of binomial ideals in [5], where the ubiquity of binomial ideals was also presented. There have been numerous publications in recent years on binomial ideals, and several of them treat the problem of the minimal generation of a binomial ideal or of the radical of it; for example, [1, 3, 6, 7, 8, 10, 12, 13, 15].

The binomial arithmetical rank of a binomial ideal $I$ (written $\operatorname{bar}(I)$ ) is the smallest integer $s$ for which there exist binomials $f_{1}, \ldots, f_{s}$ in $I$ such that $\operatorname{rad}(I)=$ $\operatorname{rad}\left(f_{1}, \ldots, f_{s}\right)$. Hence the binomial arithmetical rank is an upper bound for the arithmetical rank of a binomial ideal (written $\operatorname{ara}(I)$ ), which is the smallest integer $s$ for which there exists $f_{1}, \ldots, f_{s}$ in $I$ such that $\operatorname{rad}(I)=\operatorname{rad}\left(f_{1}, \ldots, f_{s}\right)$. From the definitions we deduce the following inequality for a binomial ideal $I$ :

$$
h(I) \leq \operatorname{ara}(I) \leq \operatorname{bar}(I) \leq \mu(I) .
$$

Received by the editors October 17, 2000 and, in revised form, January 16, 2001.

2000 Mathematics Subject Classification. Primary 14M25, 13C40, 14M10.

Key words and phrases. Affine semigroups, binomial ideals, complete intersections, settheoretic complete intersections, toric varieties. 
Here $h(I)$ denotes the height and $\mu(I)$ denotes the minimal number of generators of $I$. When $h(I)=\operatorname{ara}(I)$ the ideal $I$ is called a set-theoretic complete intersection and when $h(I)=\mu(I)$ it is called a complete intersection.

Let $K$ be a field of any characteristic and let $\bar{K}$ be the algebraic closure of $K$. Let $\mathbf{a}_{\mathbf{i}}=\left(a_{i, 1}, \ldots, a_{i, n}\right) \in \mathbb{Z}^{n}$ for $0 \leq i \leq m$ be a set $T$ of vectors of rank $n$ such that the semigroup generated by $T, \mathbb{N} T=\left\{l_{1} \mathbf{a}_{\mathbf{1}}+\cdots+l_{m} \mathbf{a}_{\mathbf{m}}: l_{1}, \ldots, l_{m} \in \mathbb{N}\right\}$, is an affine semigroup. An affine semigroup is a finitely generated semigroup with no invertible elements. Define

$$
\phi: K\left[x_{1}, \ldots, x_{m}\right] \rightarrow K\left[t_{1}, \ldots, t_{n}, t_{1}^{-1}, \ldots, t_{n}^{-1}\right]
$$

as the homomorphism of $K$-algebras for which

$$
\phi\left(x_{i}\right)=\mathbf{t}^{\mathbf{a}_{\mathbf{i}}} \quad \text { for all } i=1, \ldots, m,
$$

where $\mathbf{t}^{\mathbf{a}_{\mathbf{i}}}=t_{1}^{a_{i, 1}} \ldots t_{n}^{a_{i, n}}$. Then $\operatorname{Ker} \phi=I_{T}$ is the toric ideal of $T$ and its affine variety $V=V\left(I_{T}\right)$ of zeros in $K^{m}$ is an affine toric variety of codimension $r=m-n$, in the sense of [5], 14], which also includes non-normal varieties. $I_{T}$ is called an affine toric ideal. The image $\phi\left(K\left[x_{1}, \ldots, x_{m}\right]\right)$ is the affine semigroup ring of $T$.

Let

$$
\Phi: \mathbb{Z}^{m} \rightarrow \mathbb{Z}^{n}
$$

be the homomorphism of groups defined by

$$
\left(s_{1}, \ldots, s_{m}\right) \mapsto\left(\sum_{i=1}^{m} s_{i} \mathbf{a}_{\mathbf{i}}\right) .
$$

The elements of the lattice $\operatorname{Ker} \Phi$ are in a one-to-one correspondence with the binomials of $I_{T}$ without monomial factors: any vector $v \in \operatorname{Ker} \Phi$ is associated with the binomial in $I_{T}, F_{v}=\underline{x}^{v_{+}}-\underline{x}^{v_{-}}$, where $v_{+, i}=\max \left\{0, v_{i}\right\}, v_{-, i}=\max \left\{0,-v_{i}\right\}$ and $\underline{x}^{v_{+}}=x_{1}^{v_{+, 1}} \ldots x_{m}^{v_{+, m}}, \underline{x}^{v_{-}}=x_{1}^{v_{-, 1}} \ldots x_{m}^{v_{-, m}}$.

Let $\mathbb{Z} T=\left\{l_{1} \mathbf{a}_{\mathbf{1}}+\cdots+l_{m} \mathbf{a}_{\mathbf{m}}: l_{1}, \ldots, l_{m} \in \mathbb{Z}\right\}$ be the lattice spanned by $T$. We denote the dimension of the lattice by $\operatorname{dim}(\mathbb{Z} T)$, which in this case is equal to $n$.

We recall the definition of semigroup gluing.

Definition 1. Let $T_{1}$ and $T_{2}$ be non-empty subsets of $T$ such that $T=T_{1} \cup T_{2}$ and $T_{1} \cap T_{2}=\emptyset$. Then $T$ is called a gluing of $T_{1}$ and $T_{2}$ if there is a non-zero element $\mathbf{a} \in \mathbb{N} T_{1} \cap \mathbb{N} T_{2}$ such that $\mathbb{Z} \mathbf{a}=\mathbb{Z} T_{1} \cap \mathbb{Z} T_{2}$.

The concept of semigroup gluing was defined by J.C. Rosales in [11] and used by K. Fischer, W. Morris and J. Shapiro in 8 to characterize all complete intersection affine semigroups. They proved that for an affine semigroup $\mathbb{N} T$ which is not a free abelian semigroup it holds: $\mathbb{N} T$ is a complete intersection if and only if there are two subsets $T_{1}$ and $T_{2}$ of $T$ such that $T$ is the gluing of $T_{1}$ and $T_{2}$ and $\mathbb{N} T_{1}, \mathbb{N} T_{2}$ are complete intersection subsemigroups.

Here we also define the notion of p-gluing and inductively the notion of completely $p$-glued, which will be very important for the characterization of set-theoretic complete intersections on binomials in characteristic $p$.

Definition 2. Let $p$ be a prime number and $T_{1}$ and $T_{2}$ be non-empty subsets of $T$ such that $T=T_{1} \cup T_{2}$ and $T_{1} \cap T_{2}=\emptyset$. Then $T$ is called a $p$-gluing of $T_{1}$ and $T_{2}$ if there are an integer $k$ and a non-zero element $\mathbf{a} \in \mathbb{Z}^{n}$ such that $\mathbb{Z} T_{1} \cap \mathbb{Z} T_{2}=\mathbb{Z a}$ and $p^{k} \mathbf{a} \in \mathbb{N} T_{1} \cap \mathbb{N} T_{2}$. 
Definition 3. An affine semigroup $\mathbb{N} T$ is called completely $p$-glued if $T$ is the $p$ gluing of $T_{1}$ and $T_{2}$, where each one of the semigroups $\mathbb{N} T_{1}, \mathbb{N} T_{2}$ is completely p-glued or a free abelian semigroup.

The purpose of this paper is to prove the following results:

1. In characteristic zero the complete intersection affine toric varieties are characterized as those affine toric varieties which are set-theoretic complete intersections on binomial hypersurfaces. This result provides a new proof of the result of $[8$ and a new characterization of complete intersection affine toric varieties.

2. In positive characteristics the previous result is not true since the class of toric varieties which are set-theoretic complete intersections on binomials is larger than the one of complete intersections. We refer to [1, where we have proved that all simplicial toric varieties with full parametrization are set-theoretic complete intersections on binomials. In positive characteristic $p$, affine toric varieties which are set-theoretic complete intersections on binomial hypersurfaces are those whose underlying semigroups are completely $p$-glued. This result generalizes all known results about set theoretic complete intersection toric varieties in positive characteristic.

Throughout the paper we shall refer to the notations introduced in this section.

\section{MAin Results}

Definition 4 ([7]). A matrix $M$ with coefficients in $\mathbb{Z}$ is called mixed if every row has a positive and a negative entry. $M$ is called dominating if it does not contain any square mixed submatrix.

Theorem 1. Let $I_{T}$ be an affine toric ideal, and let $K \operatorname{er} \Phi \subset Z^{m}$ be its corresponding lattice of relations of rank $r$. Consider $r$ vectors $u_{1}, \ldots, u_{r}$ of the lattice $\operatorname{Ker} \Phi \subset Z^{m}$ and let $M$ be the matrix formed by the rows $u_{1}, \ldots, u_{r}$ and let $F_{i}$ be the binomial corresponding to the vector $u_{i}$. If $I_{T}=\operatorname{rad}\left(F_{1}, \ldots, F_{r}\right)$, then the vectors $u_{1}, \ldots, u_{r}$ are linearly independent and the matrix $M$ is mixed dominating.

Proof. Assume that the vectors $u_{1}, \ldots, u_{r}$ are linearly dependent over $\mathbb{Q}$; then they are also linearly dependent over $\mathbb{Z}$. Therefore without loss of generality we have $n u_{r}=m_{1} u_{1}+\ldots+m_{r-1} u_{r-1}$, where the coefficient $n$ of $u_{r}$ is a positive integer not divisible by the characteristic $p$ of the field $K$. By changing $u_{i}$ to $-u_{i}$, if that is necessary, we can assume that all $m_{i} \geq 0$. Using the formula $N F_{v+w}=x^{v+} F_{w}+x^{w-} F_{v}$ with $N$ some monomial we have that $N F_{n u_{r}}$ belongs to the ideal generated by $F_{u_{1}}, \ldots, F_{u_{r-1}}$. Setting $F_{u_{r}}=X-Y$, we have that

$$
N\left(X^{n-1}+X^{n-2} Y+\ldots+Y^{n-1}\right) F_{u_{r}}=B_{1} F_{u_{1}}+\ldots+B_{r-1} F_{u_{r-1}} .
$$

$F_{u_{1}}, \ldots, F_{u_{r}}$ is a regular sequence, therefore $A=N\left(X^{n-1}+X^{n-2} Y+\ldots+Y^{n-1}\right)$ as a coefficient of a syzygy belongs to the ideal generated by $F_{u_{1}}, \ldots, F_{u_{r}}$. But this is a contradiction, since the point $(1, \ldots, 1)$ is a common zero of all $F_{u_{1}}, \ldots, F_{u_{r}}$ while $A(1, \ldots, 1)=n$ is not zero. We conclude that the vectors $u_{1}, \ldots, u_{r}$ are linearly independent.

The semigroup $\mathbb{N} T$ does not have invertible elements, i.e., there is no positive integral combination of the $\mathbf{a}_{\mathbf{i}}$ 's which equals zero. Therefore each of the vectors $u_{i}$ is mixed, i.e., it has positive and negative components - by definition this means that $M$ is mixed. 
Now we prove that $M$ is dominating, i.e., no square submatrix of $M$ is mixed. The following proof is inspired by 2.3 of [7]. Assume that $N$ is a mixed $s \times s$ submatrix of $M$, with $s \geq 1$ and suppose that $s$ is maximal with respect to this property. Then up to permutations of the rows and of the variables we may assume that $N$ consists of the first $s$ lines and the first $s$ columns, so that we can write

$$
M=\left(\begin{array}{ll}
N & B \\
C & D
\end{array}\right) .
$$

In a first step we prove that if $s<r$, then $C=0$. If a row of $C$ is mixed, then we can produce a mixed $(s+1) \times(s+1)$ submatrix of $M$ adding this row and any column to $N$. Hence we may assume that all the lines of $C$ have constant sign. In this case, whenever $u_{j, i} \neq 0$, for some index $i$ and some $j>s$, then some other entry $u_{j, k}$, with $k>s$, must be non-zero of opposite sign, because the row vector $u_{j}$ is mixed. If we add the $j$ th row and the $k$ th column to $N$ we obtain a mixed $(s+1) \times(s+1)$ submatrix of $M$, which again contradicts the maximality of $s$. Hence we conclude that $s<r$ implies $C=0$.

Let $\widehat{J}=\left(F_{u}, u \in \operatorname{span}_{\mathbb{Q}}\left(u_{s+1}, \ldots, u_{r}\right) \cap \mathbb{Z}^{n}\right)$, where we set $\widehat{J}=0$ if $s=r$.

- We have that

$$
\left(F_{1}, \ldots, F_{s}\right) \subset\left(x_{1}, \ldots, x_{s}\right)
$$

because $N$ is mixed, and

$$
\left(F_{s+1}, \ldots, F_{r}\right) \subset \widehat{J}
$$

by definition. Moreover, $\widehat{J}$ is a prime ideal, so that also $\left(x_{1}, \ldots, x_{s}, \widehat{J}\right)$ is a prime ideal, since $x_{1}, \ldots, x_{s}$ do not appear in any of the generators of $\widehat{J}$. Therefore $\left(F_{1}, \ldots, F_{r}\right) \subset\left(x_{1}, \ldots, x_{s}, \widehat{J}\right)$ and, furthermore, $I=\operatorname{rad}\left(F_{1}, \ldots, F_{r}\right)$ $\subset\left(x_{1}, \ldots, x_{s}, \widehat{J}\right)$.

- Since $\widehat{J}$ is a prime ideal of height $r-s,\left(x_{1}, \ldots, x_{s}, \widehat{J}\right)$ is a prime ideal of height $r$, but $I$ is also a prime ideal of height $r$. Hence $I=\left(x_{1}, \ldots, x_{s}, \widehat{J}\right)$. In particular, since $s>0$, then $I$ contains the variable $x_{1}$, which is impossible. This completes the proof by contradiction.

Now we recall the following decomposition theorem for mixed dominating matrices (see 8]) adjusted to our notation.

Theorem 2. Let $M$ be a mixed dominating $r \times m$ matrix with $m>r>0$. Then there is a rearrangement of the rows and columns of $M$ such that the resulting matrix has the form

$$
M=\left(\begin{array}{cc}
A & 0 \\
0 & B \\
a & b
\end{array}\right)
$$

where $A, B$ are mixed dominating matrices of sizes $s \times g$ and $t \times q$ respectively with $g \geq s \geq 0, q \geq t \geq 0$ and $s+t+1=r, g+q=m$. Additionally $a$ and $b$ are non-zero, unmixed $1 \times g$ and $1 \times q$ matrices of opposite signs.

Remark 1. Up to rearrangement we can assume that $F_{1}, \ldots, F_{s}$ correspond to the first $s$ rows of $M$ and $F_{s+1}, \ldots, F_{s+t}$ correspond to the following $t$ rows of $M$. Hence we have the decomposition $T=T_{1} \cup T_{2}$, where $T_{1}=\left\{\mathbf{b}_{\mathbf{1}}, \ldots, \mathbf{b}_{\mathbf{g}}\right\}$ and 
$T_{2}=\left\{\mathbf{c}_{1}, \ldots, \mathbf{c}_{\mathbf{q}}\right\}$. It is clear that $T_{1} \cap T_{2}=\emptyset$. Denote by $y_{1}, \ldots, y_{g}$ the variables $x_{i}$ corresponding to the vectors $\mathbf{b}_{\mathbf{1}}, \ldots, \mathbf{b}_{\mathbf{g}}$, and by $z_{1}, \ldots, z_{q}$ the variables $x_{i}$ corresponding to the vectors $\mathbf{c}_{\mathbf{1}}, \ldots, \mathbf{c}_{\mathbf{q}}$.

Remark 2. Note that $I_{T_{1}}=I_{T} \cap K\left[y_{1}, \ldots, y_{g}\right]$ and $I_{T_{2}}=I_{T} \cap K\left[z_{1}, \ldots, z_{q}\right]$.

Lemma 1. With the notations introduced in Remark 1, we have that $I_{T_{1}}=$ $\operatorname{rad}\left(F_{1}, \ldots F_{s}\right)$ and $I_{T_{2}}=\operatorname{rad}\left(F_{s+1}, \ldots, F_{s+t}\right)$.

Proof. We shall prove the lemma for $I_{T_{1}}$. The binomials $F_{1}, \ldots, F_{s}$ belong to $I_{T_{1}}$, so that $\left(F_{1}, \ldots, F_{s}\right) \subset I_{T_{1}}$, whence $V\left(I_{T_{1}}\right) \subset V\left(F_{1}, \ldots, F_{s}\right)$. Suppose that the inclusion is strict; then there is a point $\left(\xi_{1}, \ldots, \xi_{g}\right)$ of $V\left(F_{1}, \ldots, F_{s}\right) \subset \bar{K}^{g}$ which is not a point of $V\left(I_{T_{1}}\right) \subset \bar{K}^{g}$. Recall that $F_{r}=M_{r}-N_{r}$, where $M_{r}$ is a monomial in the $y_{1}, \ldots, y_{g}$-variables and $N_{r}$ is a monomial in the $z_{1}, \ldots, z_{q^{-}}$ variables. Let $w=M_{r}\left(\xi_{1}, \ldots, \xi_{g}\right)$ and set $N_{r}\left(\mathbf{u}^{\mathbf{c}_{\mathbf{1}}}, \ldots, \mathbf{u}^{\mathbf{c}_{\mathbf{q}}}\right)=\mathbf{u}^{\mathbf{c}}$. Moreover let $\left(v_{1}, \ldots, v_{n}\right) \in \bar{K}^{n}$ be any root of the polynomial $u^{c}-w \in \bar{K}\left[u_{1}, \ldots, u_{n}\right]$. Then the point $\left(\xi_{1}, \ldots, \xi_{g} ; \mathbf{v}^{\mathbf{c}_{1}}, \ldots, \mathbf{v}^{\mathbf{c}_{\mathbf{q}}}\right)$ is a point of $V\left(F_{1}, \ldots, F_{s}, F_{s+1}, \ldots, F_{s+t}, F_{r}\right) \subset \bar{K}^{m}$ which is not a point of $V$. A contradiction.

Theorem 3. Let I be an affine toric ideal, and let $K \operatorname{er} \Phi \subset \mathbb{Z}^{m}$ be its corresponding lattice of relations whose rank is $r$. Suppose that $I=\operatorname{rad}\left(F_{1}, \ldots, F_{r}\right)$ where $F_{i}$ is a binomial corresponding to the vector $u_{i} \in \operatorname{Ker} \Phi \subset \mathbb{Z}^{m}$. Then up to rearrangements with respect to the notations introduced in Remark 1 we have:

- In char 0 , the semigroup $T$ is the gluing of $T_{1}$ and $T_{2}$.

- In char $p$, the semigroup $T$ is the $p$-gluing of $T_{1}$ and $T_{2}$.

Proof. Let $M$ be the matrix formed by the rows $u_{1}, \ldots, u_{r}$ and let $u_{r}=(a, b)$ be the last row of $M$, where $a=\left(\alpha_{1}, \ldots, \alpha_{g}\right), b=\left(\beta_{1}, \ldots, \beta_{q}\right)$. Assume that $\alpha_{i} \geq 0, \beta_{i} \leq 0$ for all $i$. We set

$$
\mathbf{b}=\sum_{i=1}^{g} \alpha_{i} \mathbf{b}_{\mathbf{i}}=-\sum_{i=1}^{q} \beta_{i} \mathbf{c}_{\mathbf{i}} \in \mathbb{N} T_{1} \cap \mathbb{N} T_{2} .
$$

We claim that $\mathbb{Z} T_{1} \cap \mathbb{Z} T_{2}$ is a rank one lattice generated by a, such that:

- In char $0, \mathbf{a}=\mathbf{b}$, i.e. the semigroup $T$ is the gluing of $T_{1}$ and $T_{2}$.

- In char $p, p^{k} \mathbf{a}=\mathbf{b}$ for some natural number $k$, i.e. the semigroup $T$ is the $p$-gluing of $T_{1}$ and $T_{2}$.

Let $\mathbf{a}^{\prime} \in \mathbb{Z} T_{1} \cap \mathbb{Z} T_{2}$. By definition we can write

$$
\mathbf{a}^{\prime}=\sum_{i=1}^{g} \gamma_{i} \mathbf{b}_{\mathbf{i}}=\sum_{i=1}^{q}-\gamma_{s+i} \mathbf{c}_{\mathbf{i}}
$$

for some integers $\gamma_{i}, 1 \leq i \leq g+q=m$. This implies that the vector $u=$ $\left(\gamma_{1}, \ldots, \gamma_{m}\right)$ belongs to $\operatorname{Ker} \Phi$. Let $L \subset \operatorname{Ker} \Phi$ be the lattice generated by $u_{1}, \ldots, u_{r}$. By our hypothesis both lattices are of the same rank, hence there exists some natural number $\tau$ such that $\tau \operatorname{Ker} \Phi \subset L$. Suppose $\tau$ is minimal with respect to this property. Then

$$
\tau u=\sum_{i=1}^{s} \delta_{i} u_{i}+\sum_{i=1}^{t} \delta_{s+i} u_{s+i}+\delta(a, b)
$$


for some integers $\delta_{1}, \ldots, \delta_{r-1}$ and $\delta$. Thus

$$
\begin{gathered}
\tau\left(\gamma_{1}, \ldots, \gamma_{g}\right)=\sum_{i=1}^{s} \delta_{i} u_{i}+\delta a \\
\tau\left(\gamma_{g+1}, \ldots, \gamma_{m}\right)=\sum_{i=1}^{t} \delta_{s+i} u_{s+i}+\delta b
\end{gathered}
$$

whence

$$
\tau \mathbf{a}^{\prime}=\sum_{i=1}^{g} \tau \gamma_{i} \mathbf{b}_{\mathbf{i}}=\delta \sum_{i=1}^{g} \alpha_{i} \mathbf{b}_{\mathbf{i}}=\delta \mathbf{b} .
$$

This implies that $\mathbb{Z} T_{1} \cap \mathbb{Z} T_{2}$ is a rank one $\mathbb{Z}$-module, which also contains the vector b. It follows that $\mathbb{Z} T_{1} \cap \mathbb{Z} T_{2}=\mathbb{Z} \mathbf{a}$ for some a, and there exists a natural number $\lambda$ such that $\mathbf{b}=\lambda \mathbf{a}$.

We claim that in the zero characteristic case $\lambda=1$ and in the characteristic $p$ case $\lambda=p^{k}$ for some $k$.

Suppose that our claim is not true. Then there exist a positive integer $\lambda_{1}>1$ and an integer $k$ such that $\lambda=\lambda_{1} p^{k}$ and $p$ does not divide $\lambda_{1}$, in the characteristic zero case set $p^{k}=1$. Let $\mathbf{w} \in \mathbb{Z}^{n}$; the $\mathbf{w}$-degree of an element $\mathbf{c}$ of $\mathbb{Z}^{n}$ is the dot product with $\mathbf{w}, \operatorname{deg}_{\mathbf{w}}(\mathbf{c})=\mathbf{w} \cdot \mathbf{c}$. Choose $\mathbf{w}$ such that $\operatorname{deg}_{\mathbf{w}}(\mathbf{b})>0$; for example $\mathbf{w}=\mathbf{b}$. Let $d=\operatorname{deg}_{\mathbf{w}}(\mathbf{b})$ and $d^{\prime}=\operatorname{deg}_{\mathbf{w}}(\mathbf{a})$. Then $d=\lambda d^{\prime}$. Let $\omega \in \bar{K}$ be a primitive $\lambda_{1}$-th root of unity and let $\xi \in \bar{K}$ be such that $\xi^{d^{\prime}}=\omega$. Re-arrange the coordinates of $\bar{K}^{m}$ in order to form the sequence $\left(z_{1}, \ldots, z_{g} ; w_{1}, \ldots, w_{q}\right)$. The point $\left(1, \ldots, 1 ; \xi^{\mathbf{w} \cdot \mathbf{c}_{1}}, \ldots, \xi^{\mathbf{w} \cdot \mathbf{c}_{\mathbf{q}}}\right)$ is a zero of all $F_{1}, \ldots, F_{s}$, because $(1, \ldots, 1)$ is a point of $V\left(I_{T_{1}}\right)$. It is also a zero of all $F_{s+1}, \ldots, F_{s+t}$, because $\left(\xi^{\mathbf{w} \cdot \mathbf{c}_{\mathbf{1}}}, \ldots, \xi^{\mathbf{w} \cdot \mathbf{c}_{\mathbf{q}}}\right)$ is a point of $V\left(I_{T_{2}}\right)$. Moreover $F_{r}\left(1, \ldots, 1 ; \xi^{\mathbf{w} \cdot \mathbf{c}_{1}}, \ldots, \xi^{\mathbf{w} \cdot \mathbf{c}_{\mathbf{q}}}\right)=1-\xi^{d}=0$. Thus $\left(1, \ldots, 1 ; \xi^{\mathbf{w} \cdot \mathbf{c}_{1}}, \ldots, \xi^{\mathbf{w} \cdot \mathbf{c}_{\mathbf{q}}}\right) \in V$. But $\mathbf{a} \in \mathbb{Z} T_{1} \cap \mathbb{Z} T_{2}$ means that $\mathbf{a}=\sum_{i=1}^{g} \nu_{i} \mathbf{b}_{\mathbf{i}}=$ $\sum_{i=1}^{q} \mu_{j} \mathbf{c}_{\mathbf{j}}$, for suitable integer coefficients $\nu_{i}, \mu_{j}$. Let $\nu=\left(\nu_{1}, \ldots, \nu_{g}\right)$ and $\mu=$ $\left(\mu_{1}, \ldots, \mu_{q}\right)$. Then the second equality shows that the binomial $G=z^{\nu^{+}} w^{\mu^{-}}-$ $z^{\nu^{-}} w^{\mu^{+}} \in I(V)$. Moreover $d^{\prime}=\mathbf{w} \cdot \mathbf{a}=\sum_{j=1}^{q} \mu_{j} \mathbf{w} \cdot \mathbf{c}_{\mathbf{j}}$. But then from

$$
G\left(1, \ldots, 1 ; \xi^{\mathbf{w} \cdot \mathbf{c}_{1}}, \ldots, \xi^{\mathbf{w} \cdot \mathbf{c}_{\mathbf{q}}}\right)=0
$$

we have that $0=1-\xi^{d^{\prime}}=1-\omega \neq 0$, which is a contradiction.

\section{Characterizing SET-TheOretically COMPlete interseCtions}

Theorem 4. Let $V$ be a toric variety of codimension $r$ over a field of characteristic zero. Then $\operatorname{bar}(I(V))=r$ if and only if $V$ is a complete intersection. Moreover, if $F_{1}, \ldots, F_{r}$ are binomials such that $I(V)=\operatorname{rad}\left(F_{1}, \ldots, F_{r}\right)$, then $I(V)=$ $\left(F_{1}, \ldots, F_{r}\right)$.

Proof. Suppose that $V$ is a complete intersection. Then

$$
h(I(V))=r \leq \operatorname{bar}(I(V)) \leq \mu(I(V))=r .
$$

Therefore $\operatorname{bar}(I(V))=r$.

For the converse suppose that $\operatorname{bar}(I(V))=r$. We proceed by induction on $r \geq 1$. The statement is obvious for $r=1$. Suppose that every affine toric variety of codimension smaller than $r$ whose binomial arithmetical rank is equal to the codimension is a complete intersection. 
By virtue of Theorem 3 and Remark 1 , up to rearrangements of $F_{1}, \ldots, F_{r}$ one has that $T$ is the semigroup gluing of $T_{1}$ and $T_{2}$. Note that the Krull dimension of $I_{T_{1}}$ is equal to $\operatorname{dim}\left(\mathbb{Z} T_{1}\right)$ (see [14]) and $\operatorname{bar}\left(I_{T_{1}}\right)=s$ by Lemma 1 . Therefore $h\left(I_{T_{1}}\right)=g-\operatorname{dim}\left(\mathbb{Z} T_{1}\right) \leq s$, and similarly $h\left(I_{T_{2}}\right)=q-\operatorname{dim}\left(\mathbb{Z} T_{2}\right) \leq t$. Adding the two inequalities we have $g+q-\operatorname{dim}\left(\mathbb{Z} T_{1}\right)-\operatorname{dim}\left(\mathbb{Z} T_{2}\right) \leq s+t$. From Theorem 3 we have that $T$ is the semigroup gluing of $T_{1}, T_{2}$. Therefore $g+q-\operatorname{dim}(\mathbb{Z} T)-1 \leq s+t$, from which we have $r-1 \leq r-1$, which is an equality. Therefore we started with equalities, which means that $h\left(I_{T_{1}}\right)=s$ and $h\left(I_{T_{2}}\right)=t$. By the induction hypothesis we have that $I_{T_{1}}$ is a complete intersection and $I_{T_{1}}=\left(F_{1}, \ldots, F_{s}\right)$, and also $I_{T_{2}}$ is a complete intersection and $I_{T_{2}}=\left(F_{s+1}, \ldots, F_{s+t}\right)$. By [11 semigroup gluing implies

$$
I(V)=I_{T_{1}}+I_{T_{2}}+\left(F_{r}\right)=\left(F_{1}, \ldots, F_{r}\right)
$$

and this completes the proof.

Theorem 5. Let $V$ be a toric variety of codimension $r$ over a field of characteristic $p$. Then $\operatorname{bar}(I(V))=r$ if and only if $V$ is completely p-glued.

Proof. Suppose that $\operatorname{bar}(I(V))=r$. We proceed by induction on $r \geq 1$. The statement is obvious for $r=1$. Suppose that every affine toric variety of codimension smaller than $r$ whose binomial arithmetical rank is equal to the codimension is completely $p$-glued.

By virtue of Theorem 3 and Remark 1 , up to rearrangements of $F_{1}, \ldots, F_{r}$, one has that $T$ is the semigroup $p$-gluing of $T_{1}$ and $T_{2}$. By reasoning as in Theorem 4 we have that the binomial arithmetical ranks of $I_{T_{1}}$ and $I_{T_{2}}$ are equal to their codimension, therefore by the induction hypothesis they are completely $p$-glued. This completes the proof of one implication.

For the converse, let $V$ be a toric variety of codimension $r$ over a field of characteristic $p$. Let $T$ be the semigroup defining $V$ and suppose that it is completely $p$-glued, and it is the $p$-gluing of $T_{1}$ and $T_{2}$. We shall prove that $\operatorname{bar}(I(V))=r$ by induction on $r$. For $r=1$ the claim is obvious, since $I(V)$ is a principal ideal. Therefore $\operatorname{bar}(I(V))=1$. Suppose that the claim is true for all toric varieties of codimension smaller than $r$. By hypothesis $\mathbb{Z} T_{1} \cap \mathbb{Z} T_{2}=\mathbb{Z} \mathbf{a}$ and there is an integer $k$ such that $p^{k} \mathbf{a} \in \mathbb{N} T_{1} \cap \mathbb{N} T_{2}$. Let $T_{1}=\left\{\mathbf{b}_{\mathbf{1}}, \ldots, \mathbf{b}_{\mathbf{g}}\right\}$ and $T_{2}=\left\{\mathbf{c}_{\mathbf{1}}, \ldots, \mathbf{c}_{\mathbf{q}}\right\}$. We denote by $y_{i}$, for all $i=1, \ldots, g$, the variables corresponding to the vectors of $T_{1}$ and we denote by $z_{j}$, for all $i=1, \ldots, q$, the variables corresponding to the vectors of $T_{2}$. Clearly $I_{T_{1}}=I_{T} \cap K\left[y_{1}, \ldots, y_{g}\right]$ and $I_{T_{2}}=I_{T} \cap K\left[z_{1}, \ldots, z_{q}\right]$. Since $p^{k} \mathbf{a} \in \mathbb{N} T_{1} \cap \mathbb{N} T_{2}$, we can write

$$
p^{k} \mathbf{a}=\sum \varepsilon_{i} \mathbf{b}_{\mathbf{i}}=\sum \eta_{j} \mathbf{c}_{\mathbf{j}}
$$

for some natural numbers $\varepsilon_{i}, \eta_{j}$. This implies that the binomial $F=y_{1}^{\varepsilon_{1}} \ldots y_{g}^{\varepsilon_{g}}-$ $z_{1}^{\eta_{1}} \ldots z_{q}^{\eta_{q}}=\mathbf{y}^{\varepsilon}-\mathbf{z}^{\eta}$ belongs to $I_{T}$. We claim that

$$
I_{T}=\operatorname{rad}\left(I_{T_{1}}+I_{T_{2}}+(F)\right) \text {. }
$$

The ideal $I_{T}$ is generated by binomials of the form:

$$
B=\mathbf{y}^{\gamma_{+}} \mathbf{z}^{\delta_{-}}-\mathbf{y}^{\gamma_{-}} \mathbf{z}^{\delta_{+}} .
$$

We have that $B \in I_{T}$, therefore $\sum \gamma_{+i} \mathbf{b}_{\mathbf{i}}-\sum \gamma_{-i} \mathbf{b}_{\mathbf{i}}=\sum \delta_{+j} \mathbf{c}_{\mathbf{j}}-\sum \delta_{-j} \mathbf{c}_{\mathbf{j}} \in$ $\mathbb{Z} T_{1} \cap \mathbb{Z} T_{2}=\mathbb{Z} \mathbf{a}$. Thus there exists a natural number $\tau$ such that $\sum p^{k} \gamma_{+_{i}} \mathbf{b}_{\mathbf{i}}-$ 
$\sum p^{k} \gamma_{-i} \mathbf{b}_{\mathbf{i}}=\sum p^{k} \delta_{+j} \mathbf{c}_{\mathbf{j}}-\sum p^{k} \delta_{-j} \mathbf{c}_{\mathbf{j}}=p^{k} \tau \mathbf{a}$. In particular we get the relations:

$$
\sum p^{k} \gamma_{{ }_{i}} \mathbf{b}_{\mathbf{i}}=\sum p^{k} \gamma_{-i} \mathbf{b}_{\mathbf{i}}+\sum \tau \varepsilon_{i} \mathbf{b}_{\mathbf{i}}, \quad \sum p^{k} \delta_{+_{j}} \mathbf{c}_{\mathbf{j}}=\sum p^{k} \delta_{-j} \mathbf{c}_{\mathbf{j}}+\sum \tau \eta_{j} \mathbf{c}_{\mathbf{j}} .
$$

But

$$
\begin{aligned}
B^{p^{k}}= & \mathbf{y}^{p^{k} \gamma_{+}} \mathbf{z}^{p^{k} \delta_{-}}-\mathbf{y}^{p^{k} \gamma_{-}} \mathbf{z}^{p^{k} \delta_{+}} \\
= & \left(\mathbf{y}^{p^{k} \gamma_{+}}-\mathbf{y}^{p^{k} \gamma_{-}+\tau \varepsilon}\right) \mathbf{z}^{p^{k} \delta_{-}}-\mathbf{y}^{p^{k} \gamma_{-}}\left(\mathbf{z}^{p^{k} \delta_{+}}-\mathbf{z}^{p^{k} \delta_{-}+\tau \eta}\right) \\
& +\mathbf{y}^{p^{k} \gamma_{-}} \mathbf{z}^{p^{k} \delta_{-}}\left(\mathbf{y}^{\tau \varepsilon}-\mathbf{z}^{\tau \eta}\right) .
\end{aligned}
$$

From the previous relations we get $\left(\mathbf{y}^{p^{k} \gamma_{+}}-\mathbf{y}^{p^{k} \gamma_{-}+\tau \varepsilon}\right) \in I_{T_{1}},\left(\mathbf{z}^{p^{k} \delta_{+}}-\mathbf{z}^{p^{k} \delta_{-}+\tau \eta}\right) \in$ $I_{T_{2}}$ and $\left(\mathbf{y}^{\tau \varepsilon}-\mathbf{z}^{\tau \eta}\right) \in(F)$. Therefore $B \in \operatorname{rad}\left(I_{T_{1}}+I_{T_{2}}+(F)\right)$. The claim follows by the inductive hypothesis since $V\left(I_{T_{1}}\right)$ and $V\left(I_{T_{2}}\right)$ are completely $p$-glued and their codimensions are smaller than $r$.

The cone of $T$ is the set of all positive rational combinations of elements of $T$. A ray $R$ in the cone of $T$ is an extreme ray of the cone of $T$, if given any vector $u \in R$, positive integers $\mu, c_{1}, \ldots, c_{t}$ and elements $w_{1}, \ldots, w_{t}$ of $\mathbb{N} T$ such that

$$
\mu u=c_{1} w_{1}+\ldots c_{t} w_{t}
$$

then $w_{j} \in R$ for all $j=1, \ldots, t$. In [8] it was shown that for an $n$-dimensional complete intersection affine semigroup with $n \geq 2$, its cone contains no more than $2 n-2$ extreme rays. The corresponding statement is true for semigroups such that the corresponding toric variety is a set-theoretic complete intersection on binomials.

Corollary 1. Let $\mathbb{N} T$ be an affine semigroup of rank $n$, if for some field $K$ the toric variety $V_{T}$ is a set-theoretic complete intersection on binomials and $n \geq 2$, then the cone of $T$ contains no more than $2 n-2$ extreme rays.

Proof. The proof is exactly the same as the proof of Corollary 2.4 in [8], since it was based on the fact that $\operatorname{dim}\left(\mathbb{Z} T_{1}\right)+\operatorname{dim}\left(\mathbb{Z} T_{2}\right)=\operatorname{dim}(\mathbb{Z} T)+1$, which according to Theorem 3 is true for set-theoretic complete intersections on binomials.

\section{EXAmples}

In this section we are going to present several examples to clarify the concept of $p$-gluing.

Example 1. There are examples of affine semigroups that are completely $p$-glued for every prime number $p$. From the definition and the main result of [8] we see that every complete intersection affine semigroup is completely $p$-glued for every prime $p$. But there are also examples that are not complete intersections: in [1] we have proved that over a field of positive characteristic all simplicial toric varieties with full support are set-theoretic complete intersections on binomials. Note that an $n$-dimensional toric variety is called simplicial if its cone has exactly $n$ extreme rays. It follows from Theorem 5 that every simplicial toric variety with full support is completely $p$-glued, for every $p$. In fact the same proof of Theorem 1 in [1] gives a stronger result:

Let $V$ be a simplicial toric variety of codimension $r$ over a field of characteristic $p$, such that after a suitable rearrangement of the indices it holds that $\operatorname{supp}\left(\mathbf{a}_{1}\right) \subset$ $\operatorname{supp}\left(\mathbf{a}_{2}\right) \ldots \subset \operatorname{supp}\left(\mathbf{a}_{r}\right)$. Then $V$ is completely $p$-glued for every prime $p$ and therefore it is a set-theoretic complete intersection on binomials. 
We will explicitly work out an example of this sort which was first considered by Hartshorne in [9], but the approach that we will use resembles more the one used by Moh in [10]. Consider $T$ to be $\{(d, 0),(d-1,1),(1, d-1),(0, d)\}$ and let $T_{1}=\{(d, 0),(d-1,1),(0, d)\}$ and $T_{2}=\{(1, d-1)\}$. Then the semigroup $\mathbb{N} T_{1}$ is the gluing of two free semigroups; therefore it is a complete intersection and thus completely $p$-glued for every $p$. In fact $I_{T_{1}}=\left(x_{2}^{d}-x_{1}^{d-1} x_{4}\right)$. The semigroup $\mathbb{N} T_{2}$ is free and $\mathbb{Z} T_{1} \cap \mathbb{Z} T_{2}=\mathbb{Z} T_{2}$, since $(d, 0)-(d-1,1)+(0, d)=(1, d-1)$. Also $\mathbb{N} T_{1} \cap \mathbb{N} T_{2}$ is the semigroup generated by $d(1, d-1),(d-1)(1, d-1)$ which is of rank one and isomorphic with the numerical semigroup generated by $d, d-1$. Therefore the semigroup generated by $d(1, d-1),(d-1)(1, d-1)$ contains all large multiples of $(1, d-1)$. For each prime number $p$ choose $k$ such that $p^{k}>d(d-1)-d-(d-1)$ (the Frobenius number of the semigroup generated by $d, d-1)$. Then $p^{k}(1, d-1) \in$ $\mathbb{N} T_{1} \cap \mathbb{N} T_{2}$, which means that $T$ is completely $p$-glued for every $p$. From Theorem 5 we have $V\left(I_{T}\right)$ is a set-theoretic complete intersection on binomials in positive characteristic and the two binomials are $x_{2}^{d}-x_{1}^{d-1} x_{4}$ and $x_{3}^{p^{k}}-x_{1}^{m_{1}} x_{2}^{m_{2}} x_{4}^{m_{4}}$ for appropriate $m_{1}, m_{2}, m_{4}$ which are determined by $p^{k}(1, d-1) \in \mathbb{N} T_{1} \cap \mathbb{N} T_{2}$. Note that one binomial depends on the characteristic. From Theorem 4 we have that in characteristic zero $V\left(I_{T}\right)$ is not a set-theoretic complete intersection on binomials, since $(1, d-1) \notin \mathbb{N} T_{1} \cap \mathbb{N} T_{2}$. It is a long-standing open problem to determine if $V\left(I_{T}\right)$ is a set-theoretic complete intersection in characteristic zero.

Example 2. There are examples of affine semigroups that are completely $p$-glued only for one value of $p$. For example let

$$
\begin{gathered}
T=\left\{\left(q^{s}, 0,0,0\right),\left(0, q^{s}, 0,0\right),\left(0,0, q^{s}, 0\right),\left(0,0,0, q^{s}\right)\right. \\
(1,1,0,0),(0,1,1,0),(0,0,1,1),(1,0,0,1)\}
\end{gathered}
$$

where $q$ is prime. If $T$ were completely $p$-glued for some $p$, then it would be the p-gluing of $T_{1}$ and $T_{2}$, where $\operatorname{dim}\left(\mathbb{Z} T_{1}\right)=3$ and $\operatorname{dim}\left(\mathbb{Z} T_{2}\right)=2$, or $\operatorname{dim}\left(\mathbb{Z} T_{1}\right)=4$ and $\operatorname{dim}\left(\mathbb{Z} T_{2}\right)=1$, since $\operatorname{dim}(\mathbb{Z} T)=4$ and $\operatorname{dim}\left(\mathbb{Z} T_{1} \cap \mathbb{Z} T_{2}\right)=1$. When looking at $T$ we see that the first case is impossible; therefore we should have $\operatorname{dim}\left(\mathbb{Z} T_{1}\right)=4$ and $\operatorname{dim}\left(\mathbb{Z} T_{2}\right)=1$. Note also that by [2], for simplicial affine semigroups which are $p$-glued, $\operatorname{dim}\left(\mathbb{Z} T_{1}\right)=\operatorname{dim}(\mathbb{Z} T)$ and $\operatorname{dim}\left(\mathbb{Z} T_{2}\right)=1$ always hold.

Each of the two vectors of $T$ are linearly independent; therefore $T_{2}$ has just one element. From the definition of the $p$-gluing we conclude that this element cannot be any of $\left(q^{s}, 0,0,0\right),\left(0, q^{s}, 0,0\right),\left(0,0, q^{s}, 0\right),\left(0,0,0, q^{s}\right)$, since otherwise $\mathbb{N} T_{1} \cap \mathbb{N} T_{2}=0$. By symmetry we may assume that

$$
\begin{aligned}
T_{1}=\{ & \left(q^{s}, 0,0,0\right),\left(0, q^{s}, 0,0\right),\left(0,0, q^{s}, 0\right),\left(0,0,0, q^{s}\right), \\
& (1,1,0,0),(0,1,1,0),(0,0,1,1)\}
\end{aligned}
$$

and $T_{2}=\{(1,0,0,1)\}$. Simple computations show that the semigroup generated by $T_{1}$ is a complete intersection; therefore it is completely $p$-glued for any $p$. Also $\mathbb{Z} T_{1} \cap \mathbb{Z} T_{2}=\mathbb{Z}(1,0,0,1)$, since $(1,1,0,0)-(0,1,1,0)+(0,0,1,1)=(1,0,0,1)$, and $\mathbb{N} T_{1} \cap \mathbb{N} T_{2}=\mathbb{N}\left(q^{s}, 0,0, q^{s}\right)$. Therefore $T$ is completely $p$-glued only for $p=q$. In this case we have $I_{T}=\operatorname{rad}\left(x_{5}^{q^{s}}-x_{1} x_{2}, x_{6}^{q^{s}}-x_{2} x_{3}, x_{7}^{q^{s}}-x_{3} x_{4}, x_{8}^{q^{s}}-x_{1} x_{4}\right)$.

Example 3. There are examples of affine semigroups that are never completely $p$-glued. Corollary 1 and Theorem 5 give a necessary condition for a semigroup to 
be completely $p$-glued. Therefore any $n$-dimensional affine semigroup with more than $2 n-2$ extreme rays is never completely $p$-glued.

But there are also simplicial affine semigroups that are never completely $p$-glued. For instance, in Example 2, if we replace $q^{s}$ with an integer greater than one which is not a power of a prime, with exactly the same reasoning, we get an example of a semigroup that is never completely $p$-glued. But is easy to find different examples, e.g., taking $T=\{(2,0,0),(0,2,0),(0,0,3),(1,1,0),(1,0,1),(0,1,1)\}$. As in Example 2 one can argue in the following way: if $V\left(I_{T}\right)$ were $p$-glued for some prime $p$, then by symmetry the only possible partitions for $T$ would be $T_{11}, T_{12}$ or $T_{21}, T_{22}$, where $T_{12}=(1,0,1), T_{22}=(1,1,0)$ and $T_{11}, T_{21}$ are respectively their complements with respect to $T$.

In the first case we have that $T_{11}$ and $T_{12}$ are both complete intersections but $\mathbb{Z} T_{11} \cap \mathbb{Z} T_{12}=\mathbb{Z}(1,0,1)$ and $\mathbb{N} T_{11} \cap \mathbb{N} T_{12}=\mathbb{N}(6,0,6)$, which means that $T$ is not the $p$-gluing of $T_{11}, T_{12}$ for any $p$.

In the second case we have that $\mathbb{Z} T_{21} \cap \mathbb{Z} T_{22}=\mathbb{Z}(1,1,0)$ and $\mathbb{N} T_{21} \cap \mathbb{N} T_{22}=$ $\mathbb{N}(2,2,0)$, which means that $T$ is the $p$-gluing of $T_{21}, T_{22}$ only for $p=2$. Now $T_{22}$ is a complete intersection, but $T_{21}$ is completely $p$-glued only for $p=3$, since by reasoning as before, the only possible partition of $T_{21}$ is

$$
T_{211}=\{(2,0,0),(0,2,0),(0,0,3),(1,0,1)\}
$$

and $T_{212}=\{(0,1,1)\}$, for which we have $\mathbb{Z} T_{211} \cap \mathbb{Z} T_{212}=\mathbb{Z}(0,2,2)$ and $\mathbb{N} T_{211} \cap$ $\mathbb{N} T_{212}=\mathbb{N}(0,6,6)$. Therefore in the second case as well we do not have that $T$ is completely $p$-glued for some $p$.

\section{REFERENCES}

1. M. Barile, M. Morales, A. Thoma, On Simplicial Toric varieties which are Set-Theoretic Complete Intersections, Journal of Algebra 226 (2000) 880-892. CMP 2000:11

2. M. Barile, M. Morales, A. Thoma, Set-Theoretic Complete Intersections on binomials, the simplicial toric case, Pesquimat, Universidad Nacional de San Marcos, Lima, Peru, November 2000.

3. E. Becker, R. Grobe, M. Niermann, Radicals of binomial ideals, J. Pure and Applied Algebra 117 \& 118 (1997) 41-79. MR 98k:13029

4. Ch. Delorme, Sous monoides d' intersection complete de $\mathbb{N}$, Ann. scient. Ec. Norm. Sup., 4 serie, 9 (1976) 145-154. MR 53:10821

5. D. Eisenbud and B. Sturmfels, Binomial ideals, Duke Math. J. 84 (1996) 1-45. MR 97d:13031

6. S. Eliahou and R. Villarreal, On systems of binomials in the ideal of a toric variety, LMPA 96, Laboratoire de Mathématiques Pures et Appliquées, Joseph Liouville, Calais, France, 1999.

7. K. Fischer, J. Shapiro, Mixed matrices and binomials ideals, J. Pure and Applied Algebra 113 (1996) 39-54. MR 97h:13008

8. K. Fischer, W. Morris, J. Shapiro, Affine semigroup rings that are complete intersections, Proc. Amer. Math. Soc. 125 (1997) 3137-3145. MR 97m:13026

9. R. Hartshorne, Complete Intersections in characteristic $p>0$, Amer. J. Math. 101 (1979) 380-383. MR 80d:14028

10. T. T. Moh, Set-theoretic complete intersections, Proc. Amer. Math. Soc. 94 (1985) 217-220. MR 86e: 14026

11. J. C. Rosales, On Presentations of subsemigroups of $\mathbb{N}^{n}$, Semigroup Forum 55 (1997) 152-159. MR 98h:20104

12. J.C. Rosales and P. A. Garcia-Sanchez, On complete intersection affine semigroups, Commun. Algebra 23(14) (1995) 5395-5412. MR 96m:14068

13. G. Scheja, O. Scheja, U. Storch, On regular sequences of binomials, Manuscripta Math. 98 (1999) 115-132. MR 99k:13017 
14. B. Sturmfels, Gröbner Bases and Convex Polytopes. University Lecture Series, No. 8 American Mathematical Society Providence, R.I. 1995. MR 97b:13034

15. A. Thoma, On the binomial arithmetical rank, Arch. Math. 74 (2000) 22-25. MR 2001a:14023

Dipartimento di Matematica, Università degli Studi di Bari, Via Orabona 4, 70125 BARI, ITALY

E-mail address: barile@dm.uniba.it

Université de Grenoble I, Institut Fourier, UMR 5582, B.P.74, 38402 Saint-Martin D'Hères Cedex, and IUfm de Lyon, 5 rue Anselme, 69317 Lyon Cedex, France

E-mail address: Marcel.Morales@ujf-grenoble.fr

Department of Mathematics, Purdue Univerity, West Lafayette, Indiana 47907-1395

Current address: Department of Mathematics, University of Ioannina, Ioannina 45110, Greece

E-mail address: athoma@cc.uoi.gr 